\title{
New Developments in Minimally Invasive Gynecologic Oncology Surgery
}

\author{
KATHERINE IKARD STEWART, MD,MBA and AMANDA N. FADER, MD \\ Kelly Gynecologic Oncology Service, Department of Gynecology and Obstetrics, Johns Hopkins \\ University School of Medicine, Baltimore, Maryland
}

\begin{abstract}
Minimally invasive surgery continues to transform the field of gynecologic oncology and has now become the standard of care for many early-stage malignancies. The proven benefits of minimally invasive surgery are driving the rapid introduction and dissemination of novel technologies and the increasing ability to perform even the most complex procedures less invasively. In this article, we will review the current literature on traditional multiport laparoscopy, robotic-assisted laparoscopy, laparoendoscopic single-site surgery as well as robotic-assisted laparoendoscopic single-site surgery, with a specific focus on their role in the treatment of gynecologic malignancies.
\end{abstract}

\section{Keywords}

minimally invasive surgery; laparoscopy; robotic surgery; single-site surgery; sentinel lymph node; gynecologic oncology

\section{Introduction}

Over the past 2 decades, minimally invasive surgery has become the standard of care for the treatment of many gynecologic conditions, both benign and malignant. There have been numerous studies demonstrating the benefits of minimally invasive gynecologic surgical techniques including decreased blood loss, postoperative pain, perioperative complications, shorter hospitalization, and faster recovery when compared with laparotomy. ${ }^{1,2}$ The use of less invasive technology is rapidly expanding in the field of gynecologic oncology. Between 2007 and 2011, the proportion of US patients who underwent a minimally invasive hysterectomy for endometrial cancer increased from $22 \%$ to $50 \% .^{3}$ Most importantly, oncologic outcomes for patients undergoing minimally invasive staging procedures seem comparable with open staging procedures in women with early-stage disease. ${ }^{4-6}$ As the safety and feasibility of novel minimally invasive surgical techniques are reported along with associated decreases in patient morbidity, there is a constant drive to develop newer innovations that allow for more complex procedures to be performed less invasively. However, as new technologies are disseminated, it is imperative that these techniques are

Correspondence: Amanda N. Fader, MD, Kelly Gynecologic Oncology Service, Department of Gynecology and Obstetrics, Johns Hopkins University School of Medicine, Phipps 287, Baltimore, MD. afader1@ jhmi.edu. 
critically evaluated to ensure perioperative morbidity and oncologic outcomes are at least equivalent or improved compared with traditional surgical approaches.

In this article, we will review the current literature on minimally invasive surgical innovations including traditional multiport laparoscopy (MPL), robotic-assisted laparoscopy, laparoendoscopic single-site surgery (LESS), and robotic-assisted laparoendoscopic singlesite surgery (RA-LESS), with a specific focus on their role in the treatment of gynecologic malignancies.

\section{Traditional Laparoscopy CERVICAL CANCER}

In the United States, the National Cancer Institute estimates there will be nearly 13,000 new cases of cervical carcinoma and 4100 deaths in $2016 .{ }^{7}$ Radical hysterectomy is the standard of care for the treatment of early-stage cervical carcinoma (FIGO stage IA2-IB1) and may be used in select cases for more advanced-stage disease. For decades, this procedure has been performed via laparotomy with excellent cure rates. In 1992, the first total laparoscopic radical hysterectomy with pelvic and para-aortic lymph node dissection for early-stage cervical carcinoma was reported in the literature. ${ }^{8}$ Subsequently, multiple retrospective reviews have demonstrated laparoscopic radical hysterectomy to be safe, feasible, and have a favorable morbidity profile over the traditional abdominal radical hysterectomy. ${ }^{9-16}$

In 2003, a retrospective study compared 195 patients who underwent a radical hysterectomy via an open approach with 17 patients who underwent a radical hysterectomy via a laparoscopic approach for stage I cervical cancer at Memorial Sloan Kettering. The mean estimated blood loss (693 vs. $391 \mathrm{~mL}$ ) and the mean length of stay (9.7 vs. $4.5 \mathrm{~d}$ ) were significantly less in the laparoscopic group. However, the mean operative time (296 vs. 371 min) was significantly longer in the laparoscopic group. The mean pelvic lymph node count, transfusion rate, and negative surgical margins were not significantly different between the 2 groups. There were 2 conversions to laparotomy, one due to parametrial bleeding and another secondary to pelvic adhesions and a cystotomy. ${ }^{9}$ An additional retrospective study from MD Anderson published in 2007 demonstrated similar results. The authors compared 54 patients with stage I cervical cancer who underwent laparotomy with 35 patients who underwent laparoscopic radical hysterectomy. Mean blood loss was significantly less (319 vs. $548 \mathrm{~mL}$ ) and the median length of stay was shorter (2 vs. $5 \mathrm{~d}$ ) in the laparoscopic group. The mean operating time was significantly longer in the laparoscopy group (344 vs. 307 min). Two patients required conversion to laparotomy to manage vascular injuries, the first had a trocar injury to the left external iliac vein and the second had an injury to the right external iliac vein during lymphadenectomy. The incidence of postoperative infectious morbidities including fever, wound cellulitis, urinary tract infection, pneumonia, intraabdominal abscess, and necrotizing fasciitis was significantly higher in the laparotomy group (53\% vs. $18 \%) .{ }^{10}$ No long-term follow-up data were reported.

Long-term follow-up data were reported in 2011 by Nam and colleagues who reviewed 263 patients who underwent laparoscopic radical hysterectomy for stage IA2-IIA cervical cancer that were matched 1:1 with patients who underwent open radical hysterectomy. The 5-year 
recurrence-free survival rates were equal in the laparoscopy and laparotomy groups (92.8\% and $94.4 \%$, respectively). The mean time to recurrence in the laparotomy and laparoscopy groups was also similar at 29 and 24 months, respectively. All patients were followed for $>24$ months. In addition, they also reported a decrease in perioperative morbidity and recovery times consistent with previous studies. ${ }^{13}$ In 2012, another retrospective study reported on oncologic outcomes for a total of 118 patients with stage IA2-IIA2 cervical cancer after laparoscopic radical hysterectomy. Median follow-up was 31 months (range, 1 to $89 \mathrm{mo}$ ) with 5-year recurrence-free and overall survival rates of $90 \%$ and $89 \%$, respectively. The authors concluded that laparoscopic radical hysterectomy is safe and feasible in survival and morbidity but cited the need for larger prospective trials to support the approach. ${ }^{14}$ One of the first studies to report laparoscopy outcomes in patients with bulky early-stage IB2 and IIA2 cervical cancer was then published in 2013. The retrospective analysis included 115 laparoscopic and 118 laparotomy patients. In contrast to previous studies, there was no difference in operating time between the 2 groups. In the laparoscopic group, blood loss, time to recovery of bowel function, length of stay, and postoperative complications were all significantly decreased as compared with the laparotomy group. Similar to previous outcomes data, the 5-year disease-free survival was equivalent between the laparoscopy and laparotomy groups, $78 \%$ and $77 \%$, respectively. The overall 5-year survival rate was $83 \%$ in both groups. No differences were seen in recurrence patterns or median time to recurrence. ${ }^{12}$

A prospective nonrandomized study of 157 women with stage IA2 and stage IB1 cervical cancer was published in 2015. After exclusion of 37 patients based on propensity matching, a total of 120 patients were included in the analysis. The results were similar to previous studies and demonstrated a decrease in blood loss and shorter length of stay associated with laparoscopy. Operative time was significantly longer in the laparoscopy group (216 vs. 175 min). Complication rates and survival outcomes were equivalent between the groups. ${ }^{15}$ Additional retrospective reviews have also demonstrated 5-year disease-free survival and overall survival rates to be similar for open and laparoscopic groups for patients with earlystage cervical cancer. ${ }^{6,16}$

In sum, the studies published to date demonstrate that traditional laparoscopy is safe, feasible, and seems to be oncologically equivalent to laparotomy based on numerous retrospective and few prospective studies. There is no published, randomized controlled trial data validating the use of minimally invasive surgery in this setting. However, an ongoing international randomized study, the Laparoscopy in Cervical Cancer (LACC) Study, will hopefully provide high-quality evidence in support of minimally invasive surgery for the treatment of cervical cancer. Despite the current evidence in favor of the laparoscopic radical hysterectomy, adoption rates of this complex procedure remained somewhat limited before the dissemination of the robotic platform. ${ }^{11}$

\section{ENDOMETRIAL CANCER}

According to the National Cancer Institute, an estimated 60,000 new cases of endometrial cancer will be diagnosed in the United States in 2016. Endometrial cancer is the most common gynecologic malignancy. In addition, an estimated 10,000 women will die from the 
disease this year alone. ${ }^{7}$ In the early 1990 s, reports emerged demonstrating the feasibility of laparoscopic hysterectomy with bilateral pelvic and para-aortic lymph node staging for the treatment of early-stage endometrial cancer. ${ }^{17}$ The same group published a small retrospective analysis comparing 17 laparotomy patients to 13 laparoscopy patients who underwent laparoscopic hysterectomy, bilateral salpingo-oophorectomy, and pelvic and paraaortic lymphadenectomy, and demonstrated that utilization of the laparoscopic approach resulted in significant improvement in quality of life as evidenced by shorter hospital stay and earlier return to normal activity. Laparoscopy was also noted to be significantly less expensive in terms of hospital costs. ${ }^{18}$

In contrast to cervical cancer, sound randomized data exist establishing the safety and feasibility of the laparoscopic approach in the management of early-stage endometrial cancer. A landmark randomized controlled trial comparing laparoscopy to laparotomy for management of clinical stage I to IIA endometrial cancer was published in 2009, namely the Gynecologic Oncology Group LAP2 Study (GOG-LAP2). This trial demonstrated that laparoscopy was associated with improved short-term surgical outcomes, fewer complications, and shorter hospital stay. Of note, intraoperative complications were equivalent; however, $26 \%$ of laparoscopy patients required conversion to laparotomy with poor visualization being the most commonly cited reason $(57 \%)$, followed by disease requiring laparotomy for resection (16\%) and bleeding $(11 \%){ }^{2}$ In 2012, a subsequent analysis of the GOG-LAP2 data reported a 3-year recurrence rate of $11.39 \%$ and $10.24 \%$ in the laparoscopy and laparotomy groups, respectively. Recurrence patterns and 5-year overall survival rate were also similar for the 2 approaches, thereby establishing the oncologic safety of the minimally invasive approach for the management of endometrial cancer. ${ }^{4}$

In 2012, a Cochrane review examined overall survival and disease-free survival for women with early-stage endometrial cancer undergoing hysterectomy via laparoscopy or laparotomy. This review included a total of 8 studies, including the GOG-LAP2 data and concluded that laparoscopy is associated with lower blood loss, less operative morbidities, and postoperative complications as well as faster recovery than laparotomy. Disease-free and overall survival were similar in the 2 groups indicating that laparoscopy should be the preferred surgical modality for the treatment of early-stage endometrial cancer. ${ }^{19}$

\section{Robotics}

In 2000, the da Vinci Surgical System (Intuitive Surgical Inc., Sunnyvale, CA) became the first robotic surgical system to be approved by the US Food and Drug Administration (FDA). It was ultimately approved for use in gynecologic surgery in 2005. Since its inception in 2000, >3 million minimally invasive procedures have been performed worldwide across surgical subspecialties and >2000 platforms have been installed in the United States (www.intuitivesurgical.com/company).

Robotic surgery may alleviate several of the limitations of traditional laparoscopic surgery including lack of depth perception, camera instability, decreased range of motion, and steep learning curves. In contrast, robotic surgery offers superior visualization with 3-dimensional stereoscopic vision, camera, and instrument stabilization, wristed instruments that improve 
dexterity and allow for more natural movements of the hand and wrist, and tremor canceling software that improves surgical precision. However, these advantages come with some limitations including increased cost, possibly increased operative times, absence of haptic feedback, requirement of additional ports, limited compatible instrumentation and energy sources, risk of mechanical failure, and additional surgical training. In comparison studies, it remains a question whether robotic surgery is truly superior to traditional laparoscopy but more complex procedures that would likely have required open surgery are now being performed more often minimally invasively with the assistance of the robotic platform. Despite potential limitations, robotic surgery has been widely adopted within the gynecologic oncology community.

\section{CERVICAL CANCER}

Less than a year after the FDA approval of robotic surgery in gynecology, Boggess and colleagues demonstrated the safety and feasibility of robotic-assisted radical hysterectomy (RRH) on a worldwide webcast in 2006. The same group also published a case-control study of 51 patients who underwent RRH and compared them with 49 patients who underwent radical abdominal hysterectomy. The robotic group had significantly lower blood loss (97 vs. $417 \mathrm{~mL}$ ), mean operative time ( $211 \mathrm{vs.} 248 \mathrm{~min}$ ), and mean length of hospital stay ( 1 vs. $3.2 \mathrm{~d}$ ). Mean total lymph node counts were also significantly higher in the robotic group (34 vs. 23). The rate of postoperative complications was also lower in the robotic group, but it did not reach statistically significance. The authors concluded that robotic approach is not only feasible, but may be favorable to the traditional open approach. Of note, the surgeons did not have extensive experience in traditional laparoscopic radical hysterectomy and they concluded that it was not necessary to master the robotic approach. ${ }^{20}$ Subsequently, multiple studies have also confirmed the safety and feasibility of RRH. ${ }^{21-26}$

Magrina and colleagues was the first to publish data comparing all 3 approaches to radical hysterectomy. A total of 27 robotic cases were compared with 31 laparoscopic and 35 open historical controls. The mean operative times were 190, 220, and 167 minutes and mean blood loss was 133, 208, and $444 \mathrm{~mL}$, respectively. The mean hospital stay was 1.7, 2.4, and 3.6 days, respectively. The number of lymph nodes removed was not significantly different among the groups. The authors concluded that both robotic and laparoscopic approaches are favorable to laparotomy in terms of blood loss and length of stay. Notably, the operating times for robotic radical hysterectomy were significantly less than traditional laparoscopic radical hysterectomy, taking an average of 30 minutes less to complete the procedure. ${ }^{21} \mathrm{In}$ addition, another comparative study in 2011 from MD Anderson also reported favorable operative times for the robotic over laparoscopic approach. The authors also cited a lower risk of conversion to laparotomy in the robotic group with conversion rates of $3 \%$ and $16 \%$ for robotics and laparoscopy, respectively. However, the difference in conversion rate did not reach statistical significance ${ }^{23}$ (Table 1).

In an effort to synthesize the multitude of case series, a review was published in 2011 that included 27 studies comparing a total of 342 robotic and 914 laparoscopic radical hysterectomy cases for early-stage cervical cancer. Mean operative time, blood loss, number of lymph nodes removed, intraoperative complications, and the reoperation rates were 
similar between the groups. However, major postoperative complications occurred in $9.6 \%$ of the robotic cases as compared with 5.5\% of the laparoscopic cases $(P<0.05)$. Vaginal cuff dehiscences or abscesses accounted for $63.6 \%$ of the major complications in the robotic group and only $18 \%$ in the laparoscopic group. Fistulas were notably more common after laparoscopy, accounting for $34 \%$ of the major complications in this group. The authors concluded that both procedures are safe, feasible, and equivalent regarding surgical and short-term oncological outcomes. They also noted that given the small number of robotic cases, outcomes may continue to improve as experience surpasses the learning curve. ${ }^{27}$

Overall, the oncologic outcomes of minimally invasive radical hysterectomies seem to be equivalent to those performed via laparotomy, at least in the short term. A retrospective analysis of 71 patients published by Cantrell et al ${ }^{22}$ in 2010 concluded that both progressionfree survival and overall survival for robotic radical hysterectomy are at least equivalent to laparotomy at 3 years. Another observational cohort included 100 cervical cancer patients who underwent robotic radical hysterectomy and reported overall 5-year progression-free and disease-specific survival rates of $81.4 \%$ and $88.7 \%$, respectively. ${ }^{5}$ Despite multiple case series demonstrating advantages to the laparoscopic and robotic approaches, prospective data are sparse and no randomized controlled trials have been published. A prospective randomized control trial is currently ongoing to compare outcomes of laparoscopic and robotic radical hysterectomy with abdominal radical hysterectomy. The goal of this trial is to definitively establish the minimally invasive approach to radical hysterectomy in cervical cancer as the standard of care. ${ }^{28}$

\section{ENDOMETRIAL CANCER}

The robotic platform offers technology that allows a surgeon to overcome many of the challenges associated with traditional laparoscopy as it is easier to master and does not require advanced traditional laparoscopy skills as a prerequisite. The GOG-LAP2 study cited a high conversion rate; the introduction of robotic-assisted technology has potentially allowed more challenging cases to be performed via a minimally invasive approach with fewer conversions to laparotomy. However, more recent studies have not found the conversion rate for the laparoscopic approach to be as high as cited in the LAP2 study. ${ }^{29,30}$ The learning curve for robotics is also notably less steep than for traditional laparoscopy. Lim and colleagues examined the minimum number of cases required to achieve competence in the laparoscopic versus the robotic approaches for endometrial cancer staging. The first 122 cases performed using each approach were analyzed. For robotic surgery, 24 cases were the minimum number required to achieve proficiency as compared with 49 cases of laparoscopic surgery, indicating a steeper learning curve for laparoscopy. The authors also reported that the robotic approach was associated with lower blood loss, less intraoperative and major complications, lower conversion rate, and a shorter hospital stay than the laparoscopic approach..$^{29}$ Given these findings, it is easy to understand why many gynecologic oncologists have rapidly adopted the robotic platform for the surgical staging of endometrial cancer.

To summarize the published data, a systematic review was published in 2010 comparing the outcomes associated with robotic, laparoscopic, and open surgeries for the treatment of 
endometrial cancer. A total of 8 studies were included for a total of 589 robotic, 396 laparoscopy, and 606 laparotomy cases. Robotic surgery was associated with significantly less blood loss than both the laparoscopic and open approaches. Operative times were similar for both minimally invasive approaches but significantly longer than laparotomy. As expected, length of stay was significantly shorter for the minimally invasive approaches. Lymph node counts were similar between the 3 approaches as were the rates of surgical complications including vascular, bowel, and bladder injuries, vaginal cuff dehiscence and thromboembolic complications. The conversion rate was $9.9 \%$ for laparoscopy and $4.0 \%$ for robotics, but this difference did not reach statistical significance. ${ }^{30}$

More recently, a meta-analysis was published in 2014 by Ran and colleagues to again compare these 3 surgical modalities. A total of 22 studies were included in the analysis for a total of 4420 patients with endometrial cancer. The analysis found that robotic surgery was superior to laparotomy in terms of complications, length of stay, blood loss, and transfusions. Only in operative times was laparotomy found to be favorable. When compared with laparoscopy, robotic surgery was superior in terms of blood loss and conversion rate. Laparoscopy and robotics were found to be otherwise equivalent. The authors noted that no randomized trials are available comparing the 3 modalities; however, concluded that robotic surgery is safe, feasible, and may have some advantages over traditional laparoscopy. ${ }^{31}$

The high prevalence of obesity in the endometrial cancer patient provides an ideal setting where the advantages of robotic surgery can be appreciated as higher utilization of a minimally invasive approach and fewer potential conversions to laparotomy equates to less morbidity in a patient population who is already at high risk of complications. As a result, multiple studies have been performed to identify the optimal approach for the morbidly obese endometrial cancer patient. Seamon and colleagues performed a matched cohort study in obese patients [body mass index $(\mathrm{BMI})>30 \mathrm{~kg} / \mathrm{m}^{2}$ ] undergoing surgical staging of endometrial cancer by laparotomy (191 patients) or robotic-assisted surgery (109 patients). The mean BMI was $40 \mathrm{~kg} / \mathrm{m}^{2}$ for each group. The percentage of patients who underwent adequate lymphadenectomy and total lymph node counts were equivalent between the 2 groups. The robotic group had a lower rate of blood transfusions, less complications as well as a shorter length of stay. The conversion rate from robotics to laparotomy was $15.6 \%$. The authors concluded that robotic surgery offers a favorable morbidity profile over traditional laparotomy. ${ }^{32}$ In 2011, another study compared laparotomy with robotics in obese patients with endometrial cancer and found similar benefits. Subramaniam and colleagues compared 73 laparotomy cases with 104 robotic cases in obese women. The conversion rate was $11 \%$. The mean nodes count was similar between the 2 groups. The robotic group had significantly better outcomes in terms of blood loss, blood transfusions, hospital stay, and both wound and non-wound complications. ${ }^{33}$ From current evidence, it is clear that robotics is superior to laparotomy with regard to most surgical metrics and outcomes, with the exception of operative time. More difficult to discern is whether robotics is superior to laparoscopy in the treatment of endometrial cancer. Gehrig and colleagues performed a historic case-control study to examine outcomes in obese and morbidly obese endometrial cancer patients undergoing either robotic or laparoscopic staging and demonstrated shorter operative times, less blood loss, increased lymph node counts, and a shorter hospital stay in the robotic group. These authors concluded that the robotic platform may improve outcomes 
in this specific population. ${ }^{34}$ More recently, Chan and colleagues examined the complication rates and hospital costs associated with the 3 surgical modalities in morbidly obese patients $\left(\mathrm{BMI}>40 \mathrm{~kg} / \mathrm{m}^{2}\right.$ ) being treated for endometrial cancer using data obtained from the National Inpatient Sample. They identified 1087 morbidly obese endometrial cancer patients who underwent surgery, which comprised 562 laparotomy, 98 laparoscopic, and 422 robotic cases. Overall complication rates were $23 \%, 13 \%$, and $8 \%$ in the 3 groups, respectively. Complications included in the analysis were blood transfusions, mechanical ventilation, urinary tract injury, gastrointestinal injury, wound debridement, infection, venous thromboembolism, and lymphedema. Both the laparoscopic and robotic patients received fewer blood transfusions and had shorter hospital stays than the laparotomy patients. Median total charges associated with laparotomy, laparoscopy, and robotic surgery were $\$ 39,281$, $\$ 40,997$, and $\$ 45,030$, respectively. The authors concluded that both laparoscopic and robotic surgical modalities were favorable to open surgery in the morbidly obese population. ${ }^{35}$ Taken together, the evidence to date suggests that morbidly obese patients are good candidates for either a laparoscopic or robotic approach, depending on surgeon skill and preference (Table 2).

In 2014, a retrospective study was performed to evaluate the oncologic equivalence of the robotic and laparoscopic approaches. This analysis was performed at 2 academic centers comparing 183 robotic cases with 232 laparoscopic cases for the treatment of endometrial adenocarcinoma. Disease-free survival, overall survival, and recurrence rates were similar for the 2 groups indicating equivalent oncologic outcomes. ${ }^{36}$

Overall, the literature to date supports the use of robotics over traditional laparotomy for the comprehensive staging of endometrial cancer. Robotics and laparoscopy are deemed to be equivalent in many regards, but the robotic platform has been associated with lower blood loss and conversion rates in several retrospective studies as described above. In addition, it may have distinct advantages in morbidly obese patients, but further prospective studies and randomized controlled trials are needed. From a cost perspective, robotic surgery seems to be more expensive than laparoscopy.

\section{Ovarian Cancer}

Evidence for optimal utilization of a minimally invasive approach for ovarian cancer staging is less well defined than for endometrial or cervical cancer. Both laparoscopic and robotic surgeries are routinely used to obtain pathologic diagnosis for suspicious adnexal masses. However, in advanced stages, primary debulking surgery for epithelial ovarian cancer often requires bowel resections, extensive upper abdominal surgery and a thorough evaluation of abdominal peritoneum and bowel mesentery. This type of evaluation is often difficult to achieve through laparoscopic or robotic surgery.

In early-stage ovarian cancer, several retrospective studies have demonstrated that minimally invasive surgery is both feasible and safe. ${ }^{37-40}$ The largest study to date is a multicenter retrospective study which included 300 patients with apparent early-stage ovarian cancer who underwent laparoscopic staging. Oncologic outcomes including recurrence rate, 
disease-free survival, and overall survival were comparable with currently published data for patients with early-stage ovarian cancer managed via laparotomy. ${ }^{37}$

In advanced ovarian cancer, minimally invasive surgery is not currently recommended for primary cytoreduction. However, laparoscopic evaluation may be a useful decision-making tool to identify those patients who are at high risk for suboptimal primary debulking surgery and would thus benefit from neoadjuvant chemotherapy followed by an interval debulking surgery. ${ }^{41} \mathrm{~A}$ randomized controlled trial, namely LapOvaCa-trial, is currently ongoing to assess the role of laparoscopy in predicting the result of primary debulking surgery. ${ }^{42}$ In isolated ovarian recurrences, secondary cytoreduction via a minimally invasive approach has been shown to be safe and feasible in properly selected patients but further trials are necessary. ${ }^{41}$

\section{LESS}

Given the proven benefits of minimally invasive surgery, there is an ongoing quest to determine whether MPL can be further minimized and whether this will lead to improved outcomes for women. LESS, also known as single-port laparoscopy, has emerged as a viable alternative to traditional MPL and robotic surgery. The proposed benefits of LESS include enhanced cosmesis, decreased pain, and incisional morbidity. The main disadvantage of LESS is the lack of triangulation due to instrument proximity within a single incision. This results in poor ergonomics, instrument clashing, and reduced range of motion and extensive training and experience is required to master the technique. The concept of LESS was first described in the 1970s by Wheeless and Thompson for tubal sterilization. ${ }^{43}$ However, it took many decades for innovation in surgical instrumentation and technique to allow for complicated gynecologic procedures to be performed using the LESS approach. Fader and Escobar published the first report utilizing the LESS approach for gynecologic oncology procedures in 2009. This was a series of 13 patients who underwent a variety of procedures including extrafascial hysterectomy, endometrial, and ovarian cancer-staging procedures, pelvic lymphadenectomy, and adnexal surgeries. ${ }^{44}$ Subsequently, multiple reports have been published from various institutions establishing the safety and feasibility of LESS in gynecologic oncology. ${ }^{45-51}$

Fagotti and colleagues described the first 100 early-stage endometrial cancer cases performed by LESS in a multicentric study. The median operative time was 129 minutes (range, 45 to $321 \mathrm{~min}$ ) and median blood loss was $70 \mathrm{~mL}$ (range, 10 to $500 \mathrm{~mL}$ ). Pelvic lymphadenectomy was successfully accomplished in 48 patients and para-aortic lymphadenectomy in 27 patients. As expected, operative time and estimated blood loss were greater when lymphadenectomy was performed. There were 2 conversions. One case was converted to MPL to control paravaginal bleeding and a second case was converted to laparotomy to repair an obturator nerve injury that occurred during pelvic lymph node dissection. The authors concluded that LESS is feasible for the staging of endometrial cancer. $^{48}$

The first case of a LESS radical hysterectomy for cervical cancer was reported by Garrett and Boruta in 2012. ${ }^{51}$ More recently, a retrospective, multi-institutional report described 22 
cases of stage I cervical cancer treated with LESS radical hysterectomy and pelvic lymphadenectomy. The median operative time was 260 minutes (range, 149 to $380 \mathrm{~min}$ ) and median blood loss was $60 \mathrm{~mL}$ (range, 25 to $350 \mathrm{~mL}$ ). The median number of pelvic lymph nodes removed was 22 (range, 4 to 34 ). There were 2 conversions. One patient required conversion to MPL due to obesity and another patient required conversion to laparotomy due to an external iliac vein laceration. Surgical margins were negative in all cases. ${ }^{52}$ These case series demonstrate the feasibility of LESS in gynecologic oncology. Of note, these reports are from expert surgeons in both MPL and LESS. The learning curve is steep and the proposed benefits of LESS over MPL are still being investigated.

One of the proposed benefits of LESS is the potential for reduction in postoperative pain as compared with traditional MPL. A small number of randomized controlled trials have been performed to evaluate this question. The largest trial included 100 women who were randomized to undergo a laparoscopic-assisted vaginal hysterectomy via a LESS or MPL approach by a single surgeon. The MPL was performed using a 12-mm umbilical port and 3 additional 5-mm ports. No significant differences were reported in operative times, blood loss, time to flatus, complications, or length of stay. Two of the LESS cases were converted to MPL due to adhesive disease. The patients in the LESS group reported significantly less pain at 24 and 48 hours postoperatively and used significantly less analgesics than the MPL group. However, it is worth noting that the investigators were not blinded with respect to the surgical approach when collecting the subjective pain data so it is unclear how this bias may have affected the results. ${ }^{53}$ An additional randomized trial also demonstrated decreased postoperative pain in LESS patients. This study included 60 patients who underwent adnexal surgery via the LESS or the MPL approach. In the MPL group, a total of 4 ports were used including a $10-\mathrm{mm}$ umbilical port and three 5 -mm ports. Postoperative pain scores were measured at rest and upon valsalva at multiple intervals postoperatively (20 min, 2, 4, and 8 h). The patients in the LESS group reported significantly less pain at each interval than the patients in the MPL group and they also required less pain medication. At time of discharge, no difference in pain was reported between the 2 groups. The investigators were blinded to the surgical approach. ${ }^{54}$ These trials, albeit small, provide support for the hypothesis that LESS decreases both postoperative pain and pain medication use.

In contrast, there are also several trials that have demonstrated no significant difference in postoperative pain in LESS patients as compared with MPL patients. ${ }^{55-58} \mathrm{Jung}$ and colleagues randomized 68 hysterectomy patients to a LESS or MPL approach. Four of the patients were converted from LESS to MPL. Pain scores were similar at 6 hours postoperatively and no difference in analgesic use was observed between the 2 groups. In addition, no significant differences were seen in operative times, hospital stay, blood loss, or complications. ${ }^{55}$ Another randomized trial by Fanfani and colleagues reported on 68 patients undergoing hysterectomy via LESS or MPL. The LESS approach was associated with less pain; however, there was no difference in pain medication consumption. ${ }^{56}$ Taken together, current evidence demonstrates that the LESS approach may be associated with a reduction in postoperative pain and pain medication requirements as compared with the traditional laparoscopic approach. However, it remains unclear whether the reported statistical differences accurately reflect a clinically significant decrease in postoperative pain and analgesia use. 
Enhanced cosmesis and decreased incisional morbidity are also commonly cited advantages to LESS. One small, randomized controlled trial evaluated cosmesis as the primary outcome in 40 women undergoing hysterectomy via a LESS or MPL approach. Using a validated questionnaire, the patients assessed their cosmetic satisfaction before surgery and 1,4 , and 24 weeks after surgery. The patients who underwent a LESS procedure reported significantly higher rates of cosmetic satisfaction at all timepoints. The 2 groups did not vary in terms of demographic data, operative times, uterine weights, complication rates, hospital stay, postoperative pain scores, or analgesic use. ${ }^{59}$ In addition to enhanced cosmesis, the utilization of a single incision allows for ease of tissue extraction and theoretically may decrease morbidity associated with multiple skin incisions and trocar placement, namely injury to underlying viscera and nerves. However, these injuries are quite rare in laparoscopy and to demonstrate the potential superiority of LESS-related incisional morbidities, it would require an exorbitantly large sample size. One potential drawback to the larger fascial incision utilized in LESS is the concern for postoperative herniation. Regarding this topic, there is a paucity of data in the gynecology literature; however, a small multicentric retrospective review examined the risk of postoperative herniation in 211 patients who underwent gynecologic surgery via LESS approach. The overall reported rate of umbilical hernia was $2.4 \%$ after a median follow-up of 16 months. Of the 5 patients who developed a hernia, 4 of them had significant risk factors for fascial disruption. When these subjects were excluded from the analysis, the rate of umbilical hernia in the remaining low-risk patients was $0.5 \%$, comparable with that of MPL. ${ }^{60}$

In an effort to evaluate surgical outcomes for gynecologic surgery performed via LESS, a systematic review and meta-analysis was published in 2013, which included 6 randomized trials and 15 observational studies. A total of 899 LESS procedures and 1186 MPL procedures were pooled for analysis and no significant difference in complication rates was reported. Complications included death, conversion to laparotomy, reoperation, organ injury, vascular injury, vaginal vault dehiscence, and bleeding requiring a transfusion. Other clinical outcomes including postoperative pain, change in hemoglobin, length of stay, and cosmesis could not be pooled for analysis due to limited data and variation in reporting. ${ }^{61}$ Another single institution, prospective study reported on the perioperative and late complications of 515 LESS gynecologic surgeries performed by a single surgeon. This included 300 hysterectomies, 100 ovarian cystectomies, 17 myomectomies, 18 adnexal surgeries, and 11 uncategorized. A LESS approach was successfully completed in $95.7 \%$ of cases. Twenty cases required conversion to MPL and 2 cases were converted to laparotomy. Ovarian cystectomy was the most common case type that required conversion to MPL, with an additional port inserted in $12 \%$ of these cases. The most common complication was acute blood loss requiring a blood transfusion in $7 \%$ of patients. All transfusions were in hysterectomy cases. Excluding transfusions, the perioperative complication rate within 30 days of surgery was $1.6 \%$. Patients were followed for 6 months and late complications occurred in $0.6 \%$ of patients, which included 2 umbilical hernias and 1 vaginal vault evisceration after coitus on postoperative day $68 .{ }^{62}$

The learning curve in LESS is a notable barrier for many surgeons who are already proficient in MPL and may question the clinical significance of the proposed benefits of LESS. Inevitably, operative times will at least temporarily increase for the same procedure 
as surgeons learn a novel technique. The first 100 cases of LESS hysterectomy performed by a single surgeon were analyzed. No conversions occurred. The total operative time decreased significantly over the study period and proficiency was achieved after 40 cases. ${ }^{63}$ Another small study attempted to determine if LESS skills are truly more difficult to master for a novice than MPL skills. They randomized 20 medical interns into 2 groups and measured their performance on a peg transfer task and a dissection task using either 1 incision or 3 incisions in a box trainer. Of note, these interns had no prior experience with laparoscopy. Both groups had improvement in their final scores and a plateau in the time to complete the task was noted after the same number of attempts in each group. To reach the error plateau, it required 2 more attempts in the LESS group but the overall error rate once the plateau was achieved was lower in the LESS group. ${ }^{64}$ Despite the limitations, the study suggests that basic skills for both LESS and MPL can be achieved in a similar time frame in a laboratory setting using a box trainer. It is unclear how this may translate into surgeons in training in an operating room, but advanced MPL skills may not necessarily be a prerequisite to master LESS technique.

\section{RA-LESS}

Dissemination of traditional laparoscopy in complex gynecologic procedures was initially slow but with the advent of robotic surgery, utilization of minimally invasive surgery has markedly increased. Similarly, uptake of the LESS approach has been limited but as the robotic surgical platform advances to more easily accommodate single-site surgery, the LESS approach may follow suit. The first reported case of RA-LESS in gynecology was a risk reducing hysterectomy and bilateral salpingo-oophorectomy in women with a $B R C A$ mutation performed at the Cleveland Clinic in 2009. The authors demonstrated feasibility of the approach but cited multiple challenges in adapting a robotic system created for MPL into a LESS modality. ${ }^{65}$ In 2014, the FDA approved robotic single-site instrumentation for use in benign hysterectomy and salpingo-oophorectomy procedures. These instruments are semirigid and incorporate software that enable instrument swapping allowing the surgeon to avoid the counter-intuitive right-to-left instrument orientation that is a major obstacle in learning traditional LESS.

There is a paucity of data on this novel technique. A case-control study was published in Italy in 2013 comparing 19 patients with endometrial cancer who underwent a hysterectomy and bilateral salpingo-oophorectomy via RA-LESS to a control group of 38 patients who underwent the same procedure via a LESS approach. As expected, port placement and docking took longer for the robotic group (median, $8 \mathrm{~min}$ ) than the LESS group (median, 2 $\mathrm{min}$ ). The median estimated blood loss was also significantly higher in the robotic group with 75 versus $30 \mathrm{~mL}$. Median operative times were similar and no complications were reported in either group. The authors concluded that the differences seen were likely not clinically significant and the 2 approaches were comparable. Of note, the authors did acknowledge that their extensive experience with traditional LESS may have contributed to their lack of demonstrating superiority with the addition of robotic technology. In addition, the absence of EndoWrist technology on the RA-LESS platform was cited as another reason they failed to demonstrate an improvement in intraoperative outcomes. They did, however, appreciate the improvement in instrument conflict with the robotic software. ${ }^{66}$ Scheib and 
Fader recently reported feasibility of RA-LESS with a series of 40 patients undergoing various gynecologic surgeries for both benign and malignant conditions. A total of 3 cases required conversion to multiport techniques. One additional port was required to control uterine pedicle bleeding in one case and to assist with vaginal cuff closure in a morbidly obese patient in another case. A third case was converted to a traditional multiport robotic surgery due to morbid obesity with a BMI of $55 \mathrm{~kg} / \mathrm{m}^{2}$ and a large panniculus that rose high above the operating room table and prevented safe docking of the RA-LESS platform. The authors concluded that RA-LESS is feasible and safe in select patients. ${ }^{67}$ Another retrospective cohort examined perioperative outcomes in 100 patients undergoing hysterectomy for benign indications, of which half were performed via RA-LESS and the other half via LESS. There were a total of 13 conversions to multiport procedures, 8 in the RA-LESS group and 5 in the LESS group. This difference was not statistically significant. There were no conversions to laparotomy. Estimated blood loss and major complications were equivalent. Operative time was significantly longer in the robotic group, by an average of 24.9 minutes. The learning curve as measured by a plot of total operative time for chronological cases was noted to be steeper for the RA-LESS group. ${ }^{68}$

The feasibility of both LESS and RA-LESS in gynecologic oncology has been established when performed by expert minimally invasive surgeons. Specific to gynecologic oncology, the feasibility of RA-LESS for the treatment of both endometrial and cervical cancer with utilization of sentinel lymph node (SLN) mapping has been demonstrated at a single institution. ${ }^{69,70}$ More recently, there has been the addition of articulating instruments to the single-site robotic platform including a wristed needle driver and wristed bipolar forceps. It is yet to be known if the single-site robotic platform will prompt an increase in the use of the single-site approach similar to the positive influence multiport robotic technology had on the uptake of minimally invasive surgery in gynecologic oncology. Before widespread adoption, more studies are necessary to determine if the single-site approach is superior to traditional minimally invasive techniques.

\section{Technology for SLN Mapping}

The use of SLN biopsy techniques in the staging of cervical and endometrial cancer has demonstrated promising results in several prospective studies. ${ }^{71,72}$ The benefits of SLN mapping include an improvement in positive lymph node detection and a reduction in both intraoperative complications and postoperative morbidities including lymphedema. There are multiple types of substances that can be used including isosulfan blue, methylene blue, indocyanine green (ICG), or radioisotope. Radioisotope must be injected the day prior or morning of surgery. Timing issues for the injection to be done causes a barrier to its use. In addition, it is invisible to the surgeon and can only be detected by use of a Geiger counter. In contrast, isosulfan blue or methylene blue can be injected by the surgeon in the operating room just before the procedure and directly visualized. ICG is a newer dye, which has the unique characteristic of fluorescence in the near-infrared spectrum. To utilize the fluorescent light of ICG, the Firefly camera for the robotic platform and the Pinpoint camera for laparoscopy have been developed. Both camera systems can also be utilized in their respective single-site surgical modalities as well. These camera systems allow toggling between the normal view and the view that reveals the location of the ICG. A study by Sinno 
and colleagues reported on 71 consecutive cases of endometrial cancer or complex atypical hyperplasia that underwent robotic staging with utilization of SLN mapping with either isosulfan blue or ICG. ICG demonstrated a higher rate of bilateral mapping (78.9\%) as compared with the isosulfan blue group (42.4\%). A total of 5 patients had positive lymph nodes and all of these cases were identified using the SLN protocol. In addition, ICG also seemed to have an advantage in successful mapping in obese patients. ${ }^{73}$ ICG has also been shown to be effective in SLN detection in cervical cancer patients. A recent retrospective study by Beavis and colleagues reported on 30 patients with early-stage cervical cancer who underwent RRH and SLN mapping using ICG. Bilateral mapping was successful in $87 \%$ of patients. One or more SLN was detected in all patients and completion lymphadenectomy was performed after applying the SLN algorithm. Five patients were found to have metastatic disease all of which were detected using the SLN protocol. This small study demonstrated promising results for SLN mapping to potentially replace full lymphadenectomy ${ }^{74}$ (Table 3). More studies on the clinical utility of SLN mapping in our field are eagerly anticipated.

\section{Utilization of Minimally Invasive Surgery in Gynecologic Cancer}

The benefits of minimally invasive surgery over traditional laparotomy have been demonstrated in a multitude of studies in gynecologic oncology patients; however, many patients still fail to receive the recommended standard of care. Adoption rates of both traditional laparoscopic and robotic radical hysterectomy for the treatment of invasive cervical carcinoma have been slow. Wright and colleagues retrospectively reviewed 1894 patients who underwent radical hysterectomy for cervical cancer between 2006 and 2010 in the Perspective database. In 2006, only $2 \%$ of radical hysterectomy procedures were performed laparoscopically. By 2010, 23\% of cases were laparoscopic and $10 \%$ were robotic, whereas the rate of open radical hysterectomy had fallen from $98 \%$ to $67 \%$. Large hospitals were more likely to utilize a minimally invasive approach. ${ }^{75}$ Over the past 10 years, surgeons' opinions on the minimally invasive approach to radical hysterectomy have changed drastically. In 2004, a survey of Society of Gynecologic Oncology (SGO) members found that only $11 \%$ of members surveyed felt that the laparoscopic approach to radical hysterectomy was appropriate for the management of early-stage cervical cancer. It increased to $38 \%$ of SGO members in a follow-up survey in $2007 .{ }^{76}$ Today, the minimally invasive radical hysterectomy, either laparoscopic or robotic, has become the preferred surgical modality for the management of early-stage cervical carcinoma.

For endometrial cancer, the uptake of minimally invasive surgery has also been gradual. Fader and colleagues identified 32,560 patients using the National Inpatient Sample database from 2007 to 2011 . The overall rate of minimally invasive surgery increased from $22 \%$ to $50.8 \%$ over the study period. Minimally invasive surgery was more likely to be performed in medium and high volume endometrial cancer centers and teaching hospitals. Medicaid patients and minority patients were less likely to undergo a minimally invasive surgery. ${ }^{3}$ Another retrospective cohort study identified 9799 patients who underwent hysterectomy for nonmetastatic endometrial cancer in 2012 to 2013 via the National Inpatient Sample database. This study reported an overall utilization rate of $47.6 \%$ for minimally invasive hysterectomy. Consistent with previous evidence, high volume hospitals, urban hospitals, 
and teaching hospitals were more likely to apply a minimally invasive approach. Open surgery was more likely to be performed in minority patients and at rural hospitals. The increased risk of perioperative complications with open surgery was associated with an increase in cost per case of $\$ 1243$ when compared with minimally invasive surgery. The authors estimated that 2733 complications could have been avoided and \$19 million saved if the utilization rate of minimally invasive surgery was $80 \%$ in the study group. ${ }^{77}$

\section{Conclusions}

During the last 2 decades, minimally invasive surgical techniques have revolutionized the field of gynecologic oncology. A multitude of evidence has demonstrated the improvement in patient outcomes associated with a minimally invasive approach compared with open surgery and long-term oncologic outcomes seem to be equivalent. However, the true superiority of a specific minimally invasive surgical modality is far more difficult to discern. Both traditional laparoscopy and robotic surgery have their respective advantages, and surgeon skill and preference are often the most influential factors in determining which modality is utilized for a particular case. Operative and oncologic outcomes for the patient should ultimately be of utmost importance and as novel surgical modalities are introduced, objective evidence must be sought and published to support assimilation into the field. Single-site surgery provides an opportunity perform complex surgeries through an even less invasive approach than traditional techniques, though its value over MPL remains unproven. As evidence for use of these novel technologies continues to mature, the utility of this approach as well as the optimal patient population and case selection will be further ascertained.

The cost burden to society must also be considered along with these new technologies. It is known that minimally invasive surgery not only provides an improvement in patient outcomes but also has the potential to save money in the health care system by reducing hospital stays and perioperative complications. Using the American College of SurgeonsNational Surgical Quality Improvement Project's database (ACS NSQIP), Scalici and colleagues projected that for every $10 \%$ increase in utilization of minimally invasive surgery for endometrial cancer, a projected $\$ 2.8$ million would be saved and 41 postoperative complications would be avoided. ${ }^{78}$

In the era of exponentially increasing costs of health care, the goal of minimally invasive surgery should be to optimize patient and oncologic outcomes while also curtailing costs to society. Cost effectiveness research must continue to be pursued alongside outcomes research to achieve this balance as new surgical modalities are pioneered.

\section{References}

1. Aarts JWM, Nieboer TE, Johnson N, et al. Surgical approach to hysterectomy for benign gynaecological disease. Cochrane database Syst Rev. 2015; 8:CD003677.

2. Walker JL, Piedmonte MR, Spirtos NM, et al. Laparoscopy compared with laparotomy for comprehensive surgical staging of uterine cancer: Gynecologic Oncology Group Study LAP2. J Clin Oncol. 2009; 27:5331-5336. [PubMed: 19805679]

3. Fader AN, Weise RM, Sinno AK, et al. Utilization of minimally invasive surgery in endometrial cancer care: a quality and cost disparity. Obstet Gynecol. 2016; 127:91-100. [PubMed: 26646127] 
4. Walker JL, Piedmonte MR, Spirtos NM, et al. Recurrence and survival after random assignment to laparoscopy versus laparotomy for comprehensive surgical staging of uterine cancer: Gynecologic Oncology Group LAP2 Study. J Clin Oncol. 2012; 30:695-700. [PubMed: 22291074]

5. Hoogendam JP, Verheijen RHM, Wegner I, et al. Oncological outcome and long-term complications in robot-assisted radical surgery for early stage cervical cancer: an observational cohort study. BJOG. 2014; 121:1538-1545. [PubMed: 24735243]

6. Garabedian C, Merlot B, Bresson L, et al. Minimally invasive surgical management of early-stage cervical cancer: an analysis of the risk factors of surgical complications and of oncologic outcomes. Int J Gynecol Cancer. 2015; 25:714-721. [PubMed: 25647258]

7. Howlader, N.Noone, AM.Krapcho, M., et al., editors. SEER Cancer Statistics Review, 1975-2013 (SEER Stat Fact Sheets). Bethesda, MD: NationalCancer Institute; 2016. Available at: http:// seer.cancer.gov/csr/1975_2013

8. Nezhat CR, Burrell MO, Nezhat FR, et al. Laparoscopic radical hysterectomy with paraaortic and pelvic node dissection. Am J Obstet Gynecol. 1992; 166:864-865. [PubMed: 1532291]

9. Abu-Rustum NR, Gemignani ML, Moore K, et al. Total laparoscopic radical hysterectomy with pelvic lymphadenectomy using the argon-beam coagulator: pilot data and comparison to laparotomy. Gynecol Oncol. 2003; 91:402-409. [PubMed: 14599873]

10. Frumovitz M, dos Reis R, Sun CC, et al. Comparison of total laparoscopic and abdominal radical hysterectomy for patients with early-stage cervical cancer. Obstet Gynecol. 2007; 110:96-102. [PubMed: 17601902]

11. Wright JD, Herzog TJ, Neugut AI, et al. Comparative effectiveness of minimally invasive and abdominal radical hysterectomy for cervical cancer. Gynecol Oncol. 2012; 127:11-17. [PubMed: 22735788]

12. Park J-Y, Kim D-Y, Kim J-H, et al. Laparoscopic versus open radical hysterectomy in patients with stage IB2 and IIA2 cervical cancer. J Surg Oncol. 2013; 108:63-69. [PubMed: 23737035]

13. Nam J-H, Park J-Y, Kim D-Y, et al. Laparoscopic versus open radical hysterectomy in early-stage cervical cancer: long-term survival outcomes in a matched cohort study. Ann Oncol. 2012; 23:903-911. [PubMed: 21841155]

14. Hong JH, Choi JS, Lee JH, et al. Can laparoscopic radical hysterectomy be a standard surgical modality in stage IA2-IIA cervical cancer? Gynecol Oncol. 2012; 127:102-106. [PubMed: 22683586]

15. Ditto A, Martinelli F, Bogani G, et al. Implementation of laparoscopic approach for type B radical hysterectomy: a comparison with open surgical operations. Eur J Surg Oncol. 2015; 41:34-39. [PubMed: 25468458]

16. Toptas T, Simsek T. Total laparoscopic versus open radical hysterectomy in stage IA2-IB1 cervical cancer: disease recurrence and survival comparison. J Laparoendosc Adv Surg Tech A. 2014; 24:373-378. [PubMed: 24742012]

17. Spirtos NM, Schlaerth JB, Spirtos TW, et al. Laparoscopic bilateral pelvic and paraaortic lymph node sampoing: an evolving technique. Am J Obstet Gynecol. 1995; 173:105-111. [PubMed: 7631666]

18. Spirtos NM, Schlaerth JB, Gross GM, et al. Cost and quality-of-life analyses of surgery for early endometrial cancer: laparotomy versus laparoscopy. Am J Obstet Gynecol. 1996; 174:1795-1800. [PubMed: 8678142]

19. Galaal K, Bryant A, Fisher AD, et al. Laparoscopy versus laparotomy for the management of early stage endometrial cancer. Cochrane database Syst Rev. 2012; 9:CD006655.

20. Boggess JF, Gehrig PA, Cantrell L, et al. A case-control study of robot-assisted type III radical hysterectomy with pelvic lymph node dissection compared with open radical hysterectomy. Am J Obstet Gynecol. 2008; 199:357.e1-357.e7. [PubMed: 18928973]

21. Magrina JF, Kho RM, Weaver AL, et al. Robotic radical hysterectomy: comparison with laparoscopy and laparotomy. Gynecol Oncol. 2008; 109:86-91. [PubMed: 18279944]

22. Cantrell LA, Mendivil A, Gehrig PA, et al. Survival outcomes for women undergoing type III robotic radical hysterectomy for cervical cancer: a 3-year experience. Gynecol Oncol. 2010; 117:260-265. [PubMed: 20153886] 
23. Soliman PT, Frumovitz M, Sun CC, et al. Radical hysterectomy: a comparison of surgical approaches after adoption of robotic surgery in gynecologic oncology. Gynecol Oncol. 2011; 123:333-336. [PubMed: 21872911]

24. Persson J, Reynisson P, Borgfeldt C, et al. Robot assisted laparoscopic radical hysterectomy and pelvic lymphadenectomy with short and long term morbidity data. Gynecol Oncol. 2009; 113:185190. [PubMed: 19251308]

25. Nezhat FR, Datta MS, Liu C, et al. Robotic radical hysterectomy versus total laparoscopic radical hysterectomy with pelvic lymphadenectomy for treatment of early cervical cancer. JSLS. 2008; 12:227-237. [PubMed: 18765043]

26. Estape R, Lambrou N, Diaz R, et al. A case matched analysis of robotic radical hysterectomy with lymphadenectomy compared with laparoscopy and laparotomy. Gynecol Oncol. 2009; 113:357361. [PubMed: 19345987]

27. Kruijdenberg CBM, van den Einden LCG, Hendriks JCM, et al. Robot-assisted versus total laparoscopic radical hysterectomy in early cervical cancer, a review. Gynecol Oncol. 2011; 120:334-339. [PubMed: 21236473]

28. Obermair A, Gebski V, Frumovitz M, et al. A phase III randomized clinical trial comparing laparoscopic or robotic radical hysterectomy with abdominal radical hysterectomy in patients with early stage cervical cancer. J Minim Invasive Gynecol. 2008; 15:584-588. DOI: 10.1016/j.jmig. 2008.06.013. [PubMed: 18722970]

29. Lim PC, Kang E, Park DH. A comparative detail analysis of the learning curve and surgical outcome for robotic hysterectomy with lymphadenectomy versus laparoscopic hysterectomy with lymphadenectomy in treatment of endometrial cancer: a case-matched controlled study of the first o. Gynecol Oncol. 2011; 120:413-418. [PubMed: 21194735]

30. Gaia G, Holloway RW, Santoro L, et al. Robotic-assisted hysterectomy for endometrial cancer compared with traditional laparoscopic and laparotomy approaches: a systematic review. Obstet Gynecol. 2010; 116:1422-1431. [PubMed: 21099613]

31. Ran L, Jin J, Xu Y, et al. Comparison of robotic surgery with laparoscopy and laparotomy for treatment of endometrial cancer: a meta-analysis. PLoS One. 2014; 9:e108361. [PubMed: 25259856]

32. Seamon LG, Bryant SA, Rheaume PS, et al. Comprehensive surgical staging for endometrial cancer in obese patients: comparing robotics and laparotomy. Obstet Gynecol. 2009; 114:16-21. [PubMed: 19546753]

33. Subramaniam A, Kim KH, Bryant SA, et al. A cohort study evaluating robotic versus laparotomy surgical outcomes of obese women with endometrial carcinoma. Gynecol Oncol. 2011; 122:604607. [PubMed: 21658752]

34. Gehrig PA, Cantrell LA, Shafer A, et al. What is the optimal minimally invasive surgical procedure for endometrial cancer staging in the obese and morbidly obese woman? Gynecol Oncol. 2008; 111:41-45. [PubMed: 18694588]

35. Chan JK, Gardner AB, Taylor K, et al. Robotic versus laparoscopic versus open surgery in morbidly obese endometrial cancer patients - a comparative analysis of total charges and complication rates. Gynecol Oncol. 2015; 139:300-305. [PubMed: 26363212]

36. Cardenas-Goicoechea J, Shepherd A, Momeni M, et al. Survival analysis of robotic versus traditional laparoscopic surgical staging for endometrial cancer. Am J Obstet Gynecol. 2014; 210:160.e1-160.e11. [PubMed: 24176895]

37. Gallotta V, Ghezzi F, Vizza E, et al. Laparoscopic staging of apparent early stage ovarian cancer: results of a large, retrospective, multi-institutional series. Gynecol Oncol. 2014; 135:428-434. [PubMed: 25230214]

38. Tozzi R, Köhler C, Ferrara A, et al. Laparoscopic treatment of early ovarian cancer: surgical and survival outcomes. Gynecol Oncol. 2004; 93:199-203. [PubMed: 15047236]

39. Ghezzi F, Cromi A, Uccella S, et al. Laparoscopy versus laparotomy for the surgical management of apparent early stage ovarian cancer. Gynecol Oncol. 2007; 105:409-413. [PubMed: 17275077]

40. Nezhat FR, Ezzati M, Chuang L, et al. Laparoscopic management of early ovarian and fallopian tube cancers: surgical and survival outcome. Am J Obstet Gynecol. 2009; 200:83.e1-83.e6.

[PubMed: 19019337] 
41. Fagotti A, Perelli F, Pedone L, et al. Current recommendations for minimally invasive surgical staging in ovarian cancer. Curr Treat Options Oncol. 2016; 17:3.doi: 10.1007/s11864-015-0379-8. [PubMed: 26739150]

42. Rutten MJ, Gaarenstroom KN, Van Gorp T, et al. Laparoscopy to predict the result of primary cytoreductive surgery in advanced ovarian cancer patients (LapOvCa-trial): a multicentre randomized controlled study. BMC Cancer. 2012; 12:31.doi: 10.1186/1471-2407-12-31. [PubMed: 22264278]

43. Wheeless CR, Thompson BH. Laparoscopic sterilization. Review of 3600 cases. Obstet Gynecol. 1973; 42:751-758. [PubMed: 4270720]

44. Fader AN, Escobar PF. Laparoendoscopic single-site surgery (LESS) in gynecologic oncology: technique and initial report. Gynecol Oncol. 2009; 114:157-161. [PubMed: 19481243]

45. Escobar PF, Fader AN, Rasool N, et al. Single-port laparoscopic pelvic and para-aortic lymph node sampling or lymphadenectomy: development of a technique and instrumentation. Int J Gynecol Cancer. 2010; 20:1268-1273. [PubMed: 21119563]

46. Fader AN, Rojas-Espaillat L, Ibeanu O, et al. Laparoendoscopic single-site surgery (LESS) in gynecology: a multi-institutional evaluation. Am J Obstet Gynecol. 2010; 203:501.e1-501.e6. [PubMed: 20663487]

47. Hahn H-S, Kim Y-W. Single-port laparoscopic pelvic lymph node dissection with modified radical vaginal hysterectomy in cervical cancer. Int J Gynecol Cancer. 2010; 20:1429-1432. [PubMed: 21051989]

48. Fagotti A, Boruta DM, Scambia G, et al. First 100 early endometrial cancer cases treated with laparoendoscopic single-site surgery: a multicentric retrospective study. Am J Obstet Gynecol. 2012; 206:353.e1-353.e6. [PubMed: 22365037]

49. Fanfani F, Rossitto C, Gagliardi ML, et al. Total laparoendoscopic single-site surgery (LESS) hysterectomy in low-risk early endometrial cancer: a pilot study. Surg Endosc. 2012; 26:41-46. [PubMed: 21789644]

50. Tergas AI, Fader AN. Laparoendoscopic single-site surgery (LESS) radical hysterectomy for the treatment of early stage cervical cancer. Gynecol Oncol. 2013; 129:241-243. [PubMed: 23262206]

51. Garrett LA, Boruta DM. Laparoendoscopic single-site radical hysterectomy: the first report of LESS type III hysterectomy involves a woman with cervical cancer. Am J Obstet Gynecol. 2012; 207:518.e1-518.e2. [PubMed: 23111114]

52. Boruta DM, Fagotti A, Bradford LS, et al. Laparoendoscopic single-site radical hysterectomy with pelvic lymphadenectomy: initial multi-institutional experience for treatment of invasive cervical cancer. J Minim Invasive Gynecol. 2013; 21:394-398. [PubMed: 24161887]

53. Chen Y-J, Wang P-H, Ocampo EJ, et al. Single-port compared with conventional laparoscopicassisted vaginal hysterectomy: a randomized controlled trial. Obstet Gynecol. 2011; 117:906-912. [PubMed: 21422864]

54. Fagotti A, Bottoni C, Vizzielli G, et al. Postoperative pain after conventional laparoscopy and laparoendoscopic single site surgery (LESS) for benign adnexal disease: a randomized trial. Fertil Steril. 2011; 96:255.e2-259.e2. [PubMed: 21565338]

55. Jung YW, Lee M, Yim GW, et al. A randomized prospective study of single-port and four-port approaches for hysterectomy in terms of postoperative pain. Surg Endosc. 2011; 25:2462-2469. [PubMed: 21298522]

56. Fanfani F, Fagotti A, Gagliardi ML, et al. Minilaparoscopic versus single-port total hysterectomy: a randomized trial. J Minim Invasive Gynecol. 2013; 20:192-197. [PubMed: 23332717]

57. Li M, Han Y, Feng YC. Single-port laparoscopic hysterectomy versus conventional laparoscopic hysterectomy: a prospective randomized trial. J Int Med Res. 2012; 40:701-708. [PubMed: 22613433]

58. Hoyer-Sørensen C, Vistad I, Ballard K. Is single-port laparoscopy for benign adnexal disease less painful than conventional laparoscopy? A single-center randomized controlled trial. Fertil Steril. 2012; 98:973-979. [PubMed: 22769732]

59. Song T, Cho J, Kim T-J, et al. Cosmetic outcomes of laparoendoscopic single-site hysterectomy compared with multi-port surgery: randomized controlled trial. J Minim Invasive Gynecol. 2013; 20:460-467. [PubMed: 23541248] 
60. Gunderson CC, Knight J, Ybanez-Morano J, et al. The risk of umbilical hernia and other complications with laparoendoscopic single-site surgery. J Minim Invasive Gynecol. 2012; 19:40 45. [PubMed: 22033285]

61. Murji A, Patel VI, Leyland N, et al. Single-incision laparoscopy in gynecologic surgery: a systematic review and meta-analysis. Obstet Gynecol. 2013; 121:819-828. [PubMed: 23635683]

62. Park J-Y, Kim T-J, Kang H-J, et al. Laparoendoscopic single site (LESS) surgery in benign gynecology: perioperative and late complications of 515 cases. Eur J Obstet Gynecol Reprod Biol. 2013; 167:215-218. [PubMed: 23332141]

63. Paek J, Kim S-W, Lee S-H, et al. Learning curve and surgical outcome for single-port access total laparoscopic hysterectomy in 100 consecutive cases. Gynecol Obstet Invest. 2011; 72:227-233. [PubMed: 22067204]

64. Fransen SAF, Mertens LS, Botden SMBI, et al. Performance curve of basic skills in single-incision laparoscopy versus conventional laparoscopy: is it really more difficult for the novice? Surg Endosc. 2012; 26:1231-1237. [PubMed: 22101419]

65. Escobar PF, Fader AN, Paraiso MF, et al. Robotic-assisted laparoendoscopic single-site surgery in gynecology: initial report and technique. J Minim Invasive Gynecol. 2009; 16:589-591. [PubMed: 19589731]

66. Fagotti A, Corrado G, Fanfani F, et al. Robotic single-site hysterectomy (RSS-H) vs. laparoendoscopic single-site hysterectomy (LESS-H) in early endometrial cancer: a doubleinstitution case-control study. Gynecol Oncol. 2013; 130:219-223. [PubMed: 23583415]

67. Scheib SA, Fader AN. Gynecologic robotic laparoendoscopic single-site surgery: prospective analysis of feasibility, safety, and technique. Am J Obstet Gynecol. 2015; 212:179.e1-179.e8. [PubMed: 25088863]

68. Lopez S, Mulla ZD, Hernandez L, et al. A comparison of outcomes between robotic-assisted, single-site laparoscopy versus laparoendoscopic single site for benign hysterectomy. J Minim Invasive Gynecol. 2016; 23:84-88. [PubMed: 26321172]

69. Sinno AK, Fader AN, Tanner EJ. Single site robotic sentinel lymph node biopsy and hysterectomy in endometrial cancer. Gynecol Oncol. 2015; 137:190.doi: 10.1016/j.ygyno.2014.12.033. [PubMed: 25684218]

70. Sinno AK, Tanner EJ. Robotic laparoendoscopic single site radical hysterectomy with sentinel lymph node mapping and pelvic lymphadenectomy for cervical cancer. Gynecol Oncol. 2015; 139:387.doi: 10.1016/j.ygyno.2015.08.012. [PubMed: 26307401]

71. Ballester M, Dubernard G, Lécuru F, et al. Detection rate and diagnostic accuracy of sentinel-node biopsy in early stage endometrial cancer: a prospective multicentre study (SENTI-ENDO). Lancet Oncol. 2011; 12:469-476. [PubMed: 21489874]

72. How J, Lau S, Press J, et al. Accuracy of sentinel lymph node detection following intra-operative cervical injection for endometrial cancer: a prospective study. Gynecol Oncol. 2012; 127:332-337. [PubMed: 22910695]

73. Sinno AK, Fader AN, Roche KL, et al. A comparison of colorimetric versus fluorometric sentinel lymph node mapping during robotic surgery for endometrial cancer. Gynecol Oncol. 2014; 134:281-286. [PubMed: 24882555]

74. Beavis AL, Salazar-Marioni S, Sinno AK, et al. Sentinel lymph node detection rates using indocyanine green in women with early-stage cervical cancer. Gynecol Oncol. 2016; 143:302-306. [PubMed: 27526991]

75. Wright JD, Burke WM, Wilde ET, et al. Comparative effectiveness of robotic versus laparoscopic hysterectomy for endometrial cancer. J Clin Oncol. 2012; 30:783-791. [PubMed: 22291073]

76. Mabrouk M, Frumovitz M, Greer M, et al. Trends in laparoscopic and robotic surgery among gynecologic oncologists: a survey update. Gynecol Oncol. 2009; 112:501-505. [PubMed: 19138793]

77. Mannschreck D, Matsuno RK, Moriarty JP, et al. Disparities in surgical care among women with endometrial cancer. Obstet Gynecol. 2016; 128:526-534. [PubMed: 27500330]

78. Scalici J, Laughlin BB, Finan MA, et al. The trend towards minimally invasive surgery (MIS) for endometrial cancer: an ACS-NSQIP evaluation of surgical outcomes. Gynecol Oncol. 2015; 136:512-515. [PubMed: 25462206] 
TABLE 1

Selected Publications on Laparoscopy and Robotic Surgery in Cervical Cancer

\begin{tabular}{|c|c|c|}
\hline References & No. Patients & Findings \\
\hline Magrina et $\mathrm{al}^{21}$ & $\begin{array}{l}27_{\mathrm{RRH}} \\
31_{\mathrm{LRH}} \\
35_{\mathrm{ORH}}\end{array}$ & $\begin{array}{l}\text { First study to compare all } 3 \text { surgical approaches } \\
\text { RRH and LRH lower EBL, LOS }\end{array}$ \\
\hline Soliman et $\mathrm{al}^{23}$ & $\begin{array}{l}34_{\mathrm{RRH}} \\
31_{\mathrm{LRH}} \\
30_{\mathrm{ORH}}\end{array}$ & $\begin{array}{l}\text { RRH and LRH lower EBL and LOS } \\
\text { Lower conversion risk in RRH group but not statistically significant }\end{array}$ \\
\hline Boggess et $\mathrm{al}^{20}$ & $\begin{array}{l}51_{\mathrm{RRH}} \\
49_{\mathrm{ORH}}\end{array}$ & $\begin{array}{l}\text { RRH lower EBL, OR time and LOS } \\
\text { Complication rates } 7.8 \% \text { for RRH and } 16.4 \% \text { for ORH but difference not statistically significant }\end{array}$ \\
\hline Nam et al ${ }^{13}$ & $\begin{array}{l}263_{\mathrm{LRH}} \\
263_{\mathrm{ORH}}\end{array}$ & $\begin{array}{l}\text { LRH lower EBL, LOS, and postoperative complication rates } \\
\text { No difference in } 5 \text { y recurrence-free survival }\end{array}$ \\
\hline Hoogendam et $\mathrm{al}^{5}$ & $100_{\mathrm{RRH}}$ & $\begin{array}{l}\text { Overall } 5 \text { y progression-free and disease-specific survival and long-term complications all equivalent to } \\
\text { published laparotomy data }\end{array}$ \\
\hline
\end{tabular}

EBL indicates estimated blood loss; LOS, length of stay; LRH, laparoscopic radical hysterectomy; OR, operating room; ORH, open radical hysterectomy; RRH, robotic-assisted radical hysterectomy. 
TABLE 2

Selected Publications on Laparoscopy and Robotic Surgery in Endometrial Cancer

\begin{tabular}{lll}
\hline References & No. Patients & Findings \\
\hline Gehrig et al $^{34}$ & $\begin{array}{l}\text { 49 RA-TLH } \\
\text { 32 TLH }\end{array}$ & $\begin{array}{l}\text { Examined outcomes in morbidly obese patients } \\
\text { Robotic group had shorter OR time, lower EBL, LOS, and increase LN counts when compared with } \\
\text { laparoscopy group }\end{array}$ \\
Walker et al ${ }^{2,4}$ & $\begin{array}{l}\text { 920 TAH } \\
\text { 1696 TLH }\end{array}$ & $\begin{array}{l}\text { Landmark RCT demonstrated superiority of laparoscopy over laparotomy in endometrial cancer staging } \\
\text { TLH lower EBL, LOS, and fewer postoperative complications } \\
\text { Recurrence and 5-year survival rates similar to laparotomy }\end{array}$ \\
Gaia et al ${ }^{30}$ & $\begin{array}{l}\text { 606 TAH } \\
\text { 396 TLH }\end{array}$ & $\begin{array}{l}\text { Systematic review of 8 studies } \\
\text { RA-TLH lower EBL than TLH but otherwise 2 approaches have similar } \\
\text { Complication rates similar across all 3 approaches }\end{array}$ \\
& $\begin{array}{l}\text { 567 TAH } \\
\text { 98 TLH } \\
\text { 422 RA-TLH }\end{array}$ & $\begin{array}{l}\text { Analysis using National Inpatient Sample } \\
\text { Complication rates TAH 23\%, TLH 13\%, and RA-TLH 8\% } \\
\text { TLH and RA-TLH had fewer complications and shorter LOS than laparotomy patients } \\
\text { Robotics more expensive than laparoscopy }\end{array}$ \\
\hline
\end{tabular}

EBL indicates estimated blood loss; LN, lymph node; LOS, length of stay; RA-TLH, robotic-assisted hysterectomy; RCT, randomized controlled trial; TAH, total abdominal hysterectomy; TLH, total laparoscopic hysterectomy. 
TABLE 3

Selected Publications on SLN Mapping in Cervix and Endometrial Cancer

\begin{tabular}{|c|c|c|c|}
\hline References & No. Patients & Disease Site/Dye Type & Findings \\
\hline How et $\mathrm{al}^{72}$ & 100 & Endometrial patent blue dye+ radiotracer & $\begin{array}{l}\geq 1 \text { SLN detected in } 92 \% \text { of cases } \\
\text { Bilateral mapping rate- } 72 \% \\
11 \% \text { had LN metastases } \\
\text { Sensitivity of SLN algorithm } 89 \% \text { ( } 1 \text { false negative) }\end{array}$ \\
\hline Sinno et $\mathrm{al}^{73}$ & 71 & Endometrial ICG vs. ISB & $\begin{array}{l}\text { ICG bilateral mapping rate- }-78.9 \% \\
\text { ISB bilateral mapping rate- }-42.4 \% \\
7 \% \text { had LN metastases } \\
\text { All metastases were detected using SLN algorithm } \\
\text { ICG may be superior to ISB for SLN } \\
\text { ISB may be less effective in obese patients }\end{array}$ \\
\hline Beavis et $\mathrm{al}^{74}$ & 30 & $\begin{array}{l}\text { Cervix } \\
\text { ICG }\end{array}$ & $\begin{array}{l}\geq 1 \text { SLN detected in } 100 \% \text { of cases } \\
\text { Bilateral mapping rate }-87 \% \\
16.7 \% \text { had LN metastases } \\
\text { All metastases were detected using SLN algorithm }\end{array}$ \\
\hline
\end{tabular}

ICG indicates indocyanine green; ISB, isosulfan blue; LN, lymph node; SLN, sentinel lymph node. 